\title{
FoxM1 overexpression promotes cell proliferation and migration and inhibits apoptosis in hypopharyngeal squamous cell carcinoma resulting in poor clinical prognosis
}

\author{
YAN CHEN ${ }^{1}$, YIFEI LIU ${ }^{2}$, HAOSHENG NI $^{1}$, CHUANJIN DING $^{1}$, XIAOBO ZHANG $^{1}$ and ZHENXIN ZHANG ${ }^{1}$ \\ Departments of ${ }^{1}$ Otorhinolaryngology Head and Neck Surgery, and ${ }^{2}$ Pathology, \\ Affiliated Hospital of Nantong University, Nantong, Jiangsu 226001, P.R. China
}

Received April 20, 2017; Accepted July 21, 2017

DOI: 10.3892/ijo.2017.4094

\begin{abstract}
Forkhead box M1 (FoxM1), a member of the Fox family of transcriptional factors, is involved in the development of various human malignancies. However, the expression level of FoxM1 and its functional role in hypopharyngeal squamous cell carcinoma (HSCC) remained unclear to date. The aim of the present study was to investigate the FoxM1 expression in $63 \mathrm{HSCC}$ and 20 adjacent normal tissues, as well as to evaluate its association with the clinicopathological parameters and its diagnostic value in HSCC. To further explore the biological function of FoxM1 in vitro, siRNAs were used to knockdown the expression of FoxM1 in the HSCC cell line Fadu. The results revealed that FoxM1 protein was highly expressed in HSCC tissues and that its high expression was closely associated with HSCC tumor differentiation $(\mathrm{P}=0.004)$, tumor size $(\mathrm{P}=0.002)$, clinical stage $(\mathrm{P}=0.001)$, lymph node metastasis $(\mathrm{P}=0.002)$, treatment $(\mathrm{P}=0.045)$ and expression of the proliferation marker Ki-67 $(\mathrm{P}<0.001)$. Additionally, the elevated expression of FoxM1 in HSCC patients consistently predicted a poor survival time. Knockdown of FoxM1 expression blocked Fadu cell proliferation and promoted apoptosis, and also led to the downregulation of cyclin A1 expression. Furthermore, decreased expression of FoxM1 markedly impeded cell migration and reversed the epithelial-mesenchymal transition phenotype, as indicated by decreased expression of vimentin and increased expression of E-cadherin in Fadu cells. These results indicate that FoxM1 may act as an oncogene and serve as a therapeutic target against malignant progression in HSCC.
\end{abstract}

Correspondence to: Dr Zhenxin Zhang, Department of Otorhinolaryngology Head and Neck Surgery, Affiliated Hospital of Nantong University, Nantong, Jiangsu 226001, P.R. China

E-mail: zhangzhenxinent@ntu.edu.cn

Key words: epithelial-mesenchymal transition, Forkhead box M1, hypopharyngeal squamous cell carcinoma, immunohistochemistry

\section{Introduction}

Head and neck squamous cell carcinoma (HNSCC) is the sixth most common group of malignancies worldwide, and is generally classified into four independent types of cancer (1). Hypopharyngeal squamous cell carcinoma (HSCC), which accounts for $\sim 3-5 \%$ of all HNSCCs (2), has high rates of recurrence and poor survival rates, and is regarded as the most malignant form of HNSCC $(3,4)$. At the time of diagnosis, $\sim 80 \%$ of HSCC patients are at an advanced stage of disease. However, there is only limited understanding of the underlying molecular mechanisms that lead to a highly malignant phenotype and ultimately result in the unfavorable prognosis in HSCC patients. Thus, it is urgent to investigate the pathogenesis of HSCC, to identify new biomarkers and explore innovative treatment strategies.

Tumor progression is a multistep process that involves multiple genes, including the inactivation of tumor suppressor genes and the activation of oncogenes (5). Two important features that are directly related to the severity of tumors, including HSCC, are unlimited cellular proliferation and tumor metastasis, which can occur due to the aberrant expression of key regulators controlling cell proliferation, survival and motility (6). Forkhead box M1 (FoxM1), belongs to the Fox transcription factor family, which are characterized by the presence of a 'Forkhead box' or 'winged helix' DNA-binding domain. FoxM1 acts as a key regulator of the cell cycle by influencing the phase transitions from $\mathrm{G} 1$ to $\mathrm{S}$ and $\mathrm{G} 2$ to M $(7,8)$. In addition, a previous study showed that FoxM1 acts as a regulator of a wide range of other biological processes, including apoptosis, migration and angiogenesis (9). It has been reported that a variety of tumors, such as gastric (10), non-small cell lung cancer (NSCLC) (11) and nasopharyngeal carcinoma (NPC) (12), exhibit increased expression of FoxM1. In addition, the elevated expression of FoxM1 is reported to be closely associated with poor prognosis in patients with certain types of malignant tumors, and is regarded as an independent predictor of poor survival in various solid tumors (13-15). Additionally, it has been shown that decreased expression of FoxM1 leads to a reduction in the proliferation of breast tumor (16) and leukemia cells (17), and increased apoptosis of laryngeal squamous cell carcinoma (LSCC) cells (18). 
Crosstalk between the FoxM1/Cav-1 axis and the epithelialmesenchymal transition (EMT) was demonstrated to be a critical molecular mechanism in regulating the metastasis of pancreatic cancer (19). Furthermore, Xue et al (20) have highlighted the critical interaction of FoxM1 and SMAD3 for controlling TGF- $\beta$ signaling during breast cancer metastasis. Collectively, these findings suggest that FoxM1 may act as a promising therapeutic target in numerous types of human malignancies. To the best of our knowledge, there have so far been no reports on the expression and functional role of FoxM1 in HSCC.

The aim of the present study was to clarify the expression of FoxM1 in HSCC tissues, to determine the clinical significance of FoxM1 in primary HSCC, and to evaluate the relationship between the FoxM1 expression and the prognosis of HSCC patients. In addition, to assess the function of FoxM1 in HSCC cell lines, we examined the effects of siRNA-mediated FoxM1 suppression on the proliferation, apoptosis, migration and EMT in the human HSCC cell line Fadu in vitro.

\section{Materials and methods}

Patients and tissue samples. A total of 7 fresh primary HSCC tumor tissues and 2 adjacent normal tissue samples were resected during surgery at the Affiliated Hospital of Nantong University (Nantong, China) between March 2015 and May 2016. None of the patients had received treatment prior to surgery. In addition, we retrospectively collected biopsy samples from 63 HSCC patients with complete clinical and pathological data and who had received primary treatment in our hospital between August 2009 and August 2016, during the same period, 20 adjacent normal tissue specimens were also collected. The detailed clinical characteristics of the 63 HSCC patients are summarized in Table I. Paraffin-embedded tissue blocks were obtained and sectioned in the Department of Pathology. The follow-up time ranged from 3 to 71 months, with a median time of 22 months. The overall survival (OS) time was calculated from the date of surgery to the date of death or last follow-up. The disease-free survival (DFS) time was calculated from the date of surgery to the date of recurrence or last follow-up. The last follow-up of these patients was in November 2016. This study was approved by the ethics committee of the Affiliated Hospital of Nantong University and all patients provided written informed consent. The pathological samples were obtained from the surgically resected tissue specimens to avoid disadvantaging the health or prognosis of the patients, and the privacy of the patients' personal information was maintained.

Immunohistochemistry (IHC). Formalin-fixed and paraffinembedded sections with a thickness of $4 \mu \mathrm{m}$ were dried at $60^{\circ} \mathrm{C}$ for $8 \mathrm{~h}$, followed by dewaxing in xylene, dehydration in a graded alcohol series, and washing in double-distilled water. After pretreatment in sodium citrate buffer ( $\mathrm{pH}$ 6.0, $30 \mathrm{~min}$, at $100^{\circ} \mathrm{C}$ ) to facilitate antigen retrieval, followed by natural cooling, endogenous peroxidase activity was blocked with $0.3 \% \mathrm{H}_{2} \mathrm{O}_{2}$ (ZSGB-Bio, Beijing, China) for $15 \mathrm{~min}$ at room temperature away from light. Subsequently, the slides were incubated with $10 \%$ normal goat serum for $40 \mathrm{~min}$. Primary antibodies against FoxM1 (1:100; Bioworld Technology, Inc., Nanjing, China) and Ki-67 (1:100; Santa Cruz Biotechnology,
Table I. The relationship between FoxM1 expression and clinicopathological factors in HSCC.

\begin{tabular}{|c|c|c|c|}
\hline $\begin{array}{l}\text { Clinicopathological } \\
\text { features }\end{array}$ & $\begin{array}{l}\text { No. of } \\
\text { cases }\end{array}$ & $\begin{array}{l}\text { FoxM1 } \\
\text { expression }\end{array}$ & P-value \\
\hline Age (years) & & & 0.851 \\
\hline$<60$ & 23 & $7.39 \pm 2.78$ & \\
\hline$\geq 60$ & 40 & $7.54 \pm 3.21$ & \\
\hline Sex & & & 0.321 \\
\hline Male & 3 & $5.00 \pm 3.46$ & \\
\hline Female & 60 & $7.61 \pm 2.99$ & \\
\hline Differentiation & & & $0.004^{\mathrm{a}}$ \\
\hline High differentiation & 30 & $6.37 \pm 2.67$ & \\
\hline Poor differentiation & 33 & $8.51 \pm 3.02$ & \\
\hline Tumor size $(\mathrm{cm})$ & & & $0.002^{\mathrm{a}}$ \\
\hline$\leq 2$ & 25 & $6.08 \pm 2.70$ & \\
\hline$>2$ & 38 & $8.41 \pm 2.92$ & \\
\hline Clinical stage & & & $0.001^{\mathrm{a}}$ \\
\hline I-II & 20 & $5.65 \pm 2.73$ & \\
\hline III-IV & 43 & $8.34 \pm 2.81$ & \\
\hline Lymph node metastasis & & & $0.002^{\mathrm{a}}$ \\
\hline Negative & 30 & $6.30 \pm 2.84$ & \\
\hline Positive & 33 & $8.57 \pm 2.84$ & \\
\hline Treatment & & & $0.045^{\mathrm{b}}$ \\
\hline Surgery only & 29 & $6.64 \pm 2.91$ & \\
\hline Surgery and radiation & 19 & $7.74 \pm 2.71$ & \\
\hline $\begin{array}{l}\text { Surgery, radiation, } \\
\text { chemotherapy }\end{array}$ & 12 & $8.18 \pm 3.42$ & \\
\hline Other treatment & 3 & $11.33 \pm 1.15$ & \\
\hline Ki-67 expression & & & $<0.001^{\mathrm{a}}$ \\
\hline Low expression & 26 & $5.35 \pm 2.01$ & \\
\hline High expression & 37 & $8.99 \pm 2.73$ & \\
\hline
\end{tabular}

${ }^{\mathrm{a}} \mathrm{P}<0.01 ;{ }^{\mathrm{b}} \mathrm{P}<0.05$.

Inc., Dallas, TX, USA) were applied overnight in a moist chamber at $4^{\circ} \mathrm{C}$. After $20 \mathrm{~h}$, the slides were washed three times with phosphate-buffered saline (PBS), then were incubated with a 2-step Plus Poly-HRP Anti-Mouse/Rabbit IgG detection system (ZSGB-Bio) at room temperature for $30 \mathrm{~min}$. 3,3'-diaminobenzidine (DAB) (ZSGB-Bio) was used for the visualization of immunoreactivity, which appeared as a pale brown color. Nuclei were stained blue with hematoxylin.

Immunostained tissue sections were reviewed and scored independently by two pathologists without knowledge of the patient characteristics. The percentage of staining and the staining intensity were recorded as follows: $0(0-4 \%), 1$ (5-25\%), 2 (26-50\%), 3 (51-75\%) and 4 (76-100\%); and 0 (negative), 1 (weak), 2 (moderate) and 3 (strong). The products of these two scores were used as the final staining scores (ranging from 0 to 12). According to the final score, the expression levels of FoxM1 or Ki-67 were categorized as low $(<6)$ or high $(\geq 6)$. 
Cell culture and siRNA transfection. The HSCC cell line Fadu was purchased from the ATCC (Shanghai, China) and cultured in Dulbecco's modified Eagle's medium (DMEM; GE Healthcare Life Sciences-HyClone Laboratories, Logan, UT, USA) supplemented with $10 \%$ fetal bovine serum (FBS; Gibco-Thermo Fisher Scientific, Cambridge, MA, USA), in a humidified incubator with $5 \% \mathrm{CO}_{2}$ at $37^{\circ} \mathrm{C}$.

Small interfering RNAs (siRNAs) targeting FoxM1 and a control siRNA were obtained from Biomics Biotechnologies, Co., Ltd. (Nantong, China). Fadu cells were placed in 96- or 6 -well plates in complete DMEM overnight. When the cells reached $\sim 50 \%$ confluence, they were transfected with the siRNAs using Lipofectamine 2000 (Invitrogen, Carlsbad, CA, USA) according to the manufacturer's instructions. After $6 \mathrm{~h}$ of transfection, the medium containing siRNAs and Lipofectamine 2000 was replaced with $100 \mu$ l (96-well plates) or $2 \mathrm{ml}$ (6-well plates) of complete DMEM. RNAs and proteins were obtained at 48 and $72 \mathrm{~h}$ after transfection, respectively. The sequences of FoxM1 siRNAs (si-FoxM1) were as follows: \#1, 5'-GGAAAUGCUUGUGAUUCAAdTdT-3' (sense) and 5'-UUGAAUCACAAGCAUUUCCdTdT-3' (antisense); \#2, 5'-GGAUGUGAAUCUUCCUAGAdTdT-3' (sense) and 5'-UC UAGGAAGAUUCACAUCCdTdT-3' (antisense); \#3, 5'-CCAA CAGGAGUCUAAUCAdTdT-3' (sense) and 5'-UUGAUUAG ACUCCUGUUGGdTdT-3' (antisense); and \#4, 5'-GGAUUUC AGCCCAGUACAAdTdT-3' (sense) and 5'-UUGUACUGGG UGAAAUCCdTdT-3' (antisense). The sequences of the negative control siRNA (si-NC) were 5'-UUCUCCGAACGUGU CACGUdTdT-3' (sense) and 5'-ACGUGACACGUUCGGAG AAdTdT-3' (antisense).

Reverse transcription-quantitative PCR (RT-qPCR). Total RNA was extracted from cultured cells using TRIzol ${ }^{\circledR}$ reagent (Vazyme, Nanjing, China) and reverse transcribed into complementary DNA (cDNA) using a cDNA synthesis kit (Roche Diagnostics GmbH, Mannheim, Germany) according to the manufacturer's instructions. qPCR was performed using the AceQ ${ }^{\circledR}$ qPCR SYBR Green-Master Mix kit (Vazyme). The primer sequences were as follows: Human GAPDH, 5'-GAAGGTGAAGGTCGGAGTC-3' (forward) and 5'-GAAGATGGTGATGGGATTTC-3' (reverse); and FoxM1, 5'-CAGACTATCAAGGAGGAAG-3' (forward) and 5'-CCAGGAGTGAGATGATTC-3' (reverse). The qPCR conditions were $95^{\circ} \mathrm{C}$ for $10 \mathrm{~min}$, followed by 40 cycles of $95^{\circ} \mathrm{C}$ for $15 \mathrm{sec}, 60^{\circ} \mathrm{C}$ for $30 \mathrm{sec}$ and $72^{\circ} \mathrm{C}$ for $30 \mathrm{sec}$. The levels of FoxM1 mRNA were quantified using the $2^{-\Delta \Delta \mathrm{CT}}$ method and normalized against the levels of GAPDH.

Protein extraction and western blot analysis. Tissue and cell samples were lysed in lysis buffer (PMSF: RIPA, 1:100; Beyotime Institute of Biotechnology, Haimen, China). Protein concentrations were quantified using the bicinchoninic acid (BCA) protein assay method, and $20 \mu \mathrm{g}$ protein samples were separated by sodium dodecyl sulfate-polyacrylamide gel electrophoresis (SDS-PAGE) and electroblotted onto PVDF membranes (Millipore, Bedford, MA, USA). After blocking in $5 \%$ skimmed milk in Tris-buffered saline Tween-20 (TBST) for $2 \mathrm{~h}$, the membranes were incubated with the primary antibodies overnight at $4^{\circ} \mathrm{C}$ [anti-FoxM1 (1:1,000; Bioworld Technology), anti-GAPDH (1:8,000; Abways Technology,
Wynne, AR, USA), anti-proliferating cell nuclear antigen (PCNA) (\#ab2426, 1:2,000; Abcam), anti-cyclin A1 (\#sc-751; 1:300; Santa Cruz Biotechnology), anti-E-cadherin (\#RLT1453, 1:300; Suzhou Ruiying Biological Technology, Co., Ltd., Jiaozuo, China) and anti-vimentin (\#RLT4879, 1:300; Suzhou Ruiying Biological Technology)], followed by incubation for $1 \mathrm{~h}$ with the corresponding secondary antibody $(1: 1,000)$ at room temperature. An ECL Plus kit (ZSbio, Beijing, China) was used to detect the immunoreactive bands.

Cell Counting kit-8(CCK-8) cell proliferation assay. Fadu cells were seeded into a 96 -well plate at a density of $5 \times 10^{3}$ cells/well and cultured in complete medium overnight. Subsequently, the cells were transfected with FoxM1-siRNAs or control siRNA. After $6 \mathrm{~h}$ of transfection, the medium was replaced with $100 \mu \mathrm{l}$ of complete DMEM containing $10 \mu \mathrm{lCCK}-8$ solution (CCK-8 kit; Beyotime Institute of Biotechnology) and the cells were incubated for $1.5 \mathrm{~h}$. The end of this incubation was defined as $0 \mathrm{~h}$, and other subsequent time-points $(6,12,24,48$ and $72 \mathrm{~h}$ ) were also analyzed. The optical density (OD) value of each well was measured at $450 \mathrm{~nm}$ using a microplate reader (Hitachi, Ltd., Tokyo, Japan).

Flow cytometric analyses of the cell cycle and cell apoptosis. Cells were serum-deprived for $72 \mathrm{~h}$, followed by incubation with complete medium for $6,12,24$ and $36 \mathrm{~h}$; cells were collected at every time-point. The cells were then seeded into a 6-well plate and cultured overnight, then transfected with si-FoxM1 or si-NC. After $48 \mathrm{~h}$, the cells were collected and fixed in $70 \%$ ice-cold ethylalcohol (precooled at $4^{\circ} \mathrm{C}$ ) for at least $24 \mathrm{~h}$ at $-20^{\circ} \mathrm{C}$. Following centrifugation, the cells were washed with ice-cold PBS three times to remove the fixation fluid. Subsequently, with the addition of $1 \mathrm{ng} / \mathrm{ml}$ RNaseA, the cells were incubated in the dark for $20 \mathrm{~min}$ at $4^{\circ} \mathrm{C}$. Propidium iodide (PI; Becton-Dickinson, San Jose, CA, USA) was then added for staining. Finally, cell cycle distribution was analyzed with a FACSCalibur flow cytometer (Becton-Dickinson) and CellQuest acquisition and analysis programs (BectonDickinson). Each experiment was performed in triplicate.

For the analysis of apoptosis, cells were double-stained with an Annexin V-FITC/PI apoptosis detection kit (BBI) according to the manufacturer's protocol and analyzed using the FACSCalibur flow cytometer.

Wound healing assay. Fadu cells were seeded into a 6-well plate and transfected with si-FoxM1 or si-NC. After $24 \mathrm{~h}$, cell confluence reached $90 \%$ and wounds were created by scraping the cells with a $100 \mu 1$ pipette tip. Subsequently, 1X PBS was used to remove the free-floating cells and serum-free medium was added to the 6 -well plate. After $48 \mathrm{~h}$, a microscope (Olympus, Tokyo, Japan) was used to observe the migrated distance of the cells. Duplicate wells of the same treatment groups were examined.

Immunofluorescence analysis. The expression of FoxM1,EMT markers and $\beta$-tubulin were observed by immunostaining and imaging. After transfection with si-FoxM1 or si-NC for $48 \mathrm{~h}$, cells were seeded on coverslips, which were pre-placed in a 24 -well plate and fixed with $4 \%$ paraformaldehyde after $24 \mathrm{~h}$ adherent growth. After $40 \mathrm{~min}$, cells were washed with 

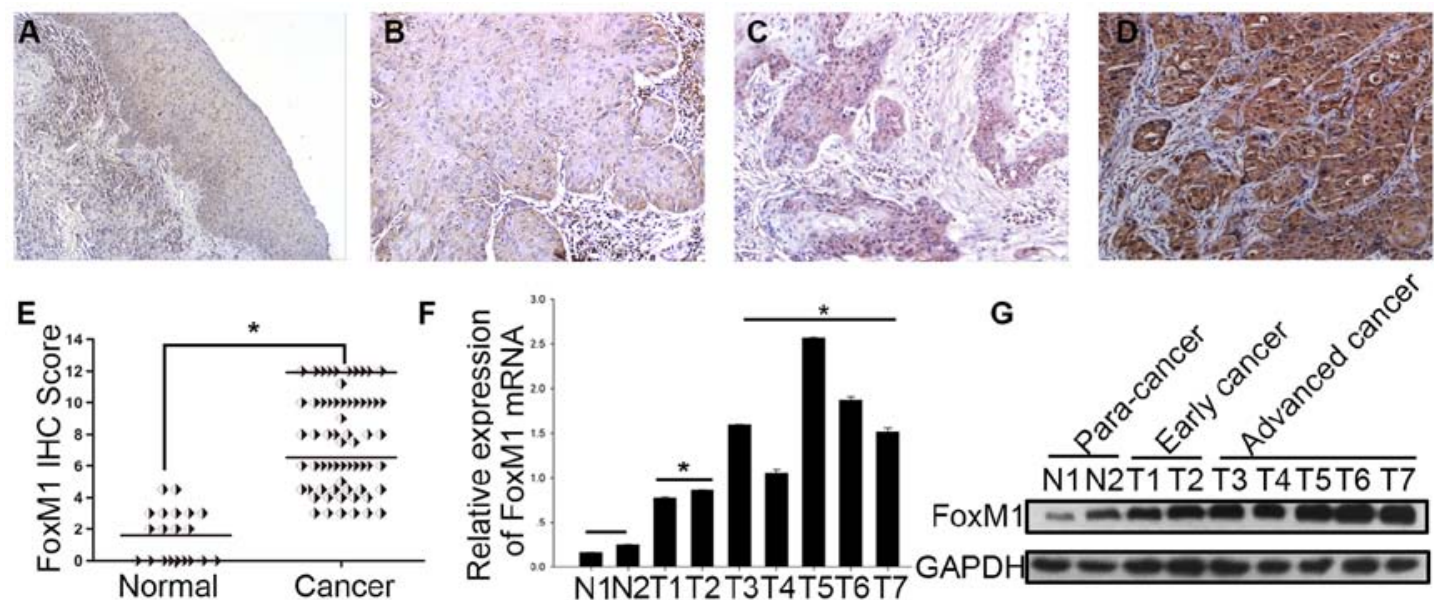

G

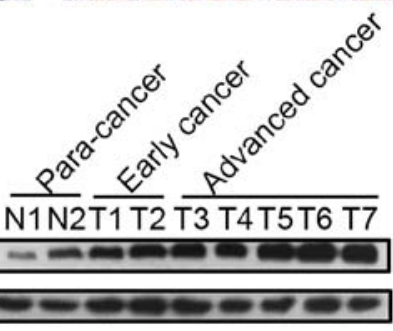

Figure 1. Relative FoxM1 expression levels in HSCC and adjacent normal tissues. (A-D) Representative immunohistochemical staining of FoxM1 in HSCC tumor tissues and adjacent normal tissues (magnification, x200). (A) Negative FoxM1 staining in normal tissues; (B) weak, (C) moderate and (D) strong positive FoxM1 staining in HSCC tissues. (E) The immunostaining intensity of FoxM1 was significantly higher in HSCC specimens compared with that in normal tissues $(\mathrm{P}<0.001)$. (F and G) RT-qPCR and western blotting were used to detect the relative expression levels of FoxM1 in fresh HSCC and para-cancer tissue samples. ${ }^{*} \mathrm{P}<0.05$.

1X PBS and blocked in Immunol Staining Blocking Buffer (\#P0102; Beyotime Institute of Biotechnology) for $2 \mathrm{~h}$ at room temperature. Cells were incubated with primary antibodies against FoxM1 (1:100; Bioworld Technology), cyclin A1 (\#sc-751, 1:100; Santa Cruz Biotechnology), PCNA (\#ab2426, 1:100; Abcam), E-cadherin (\#RLT1453, 1:100; Suzhou Ruiying Biological Technology) vimentin (\#RLT4879, 1:100; Suzhou Ruiying Biological Technologyl) and $\beta$-tubulin (\#RLM3030, 1:100; Suzhou Ruiying Biological Technology) for $20 \mathrm{~h}$, and then incubated with Alexa Fluor-conjugated secondary antibodies (1:1000; Invitrogen/Thermo Fisher Scientific) and Hoechst stain (Sigma-Aldrich, St. Louis, MO, USA) for $2 \mathrm{~h}$ at room temperature in the dark. The images were viewed and recorded with a fluorescence microscope (Leica Microsystems, Wetzlar, Germany).

Immunocytochemical staining. Cells were seeded on coverslips that were pre-placed in a 24-well plate and treated with si-FoxM1or si-NC for $72 \mathrm{~h}$. The cells were then fixed with $4 \%$ paraformaldehyde for $40 \mathrm{~min}$ and incubated with $4 \%$ BSA for $2 \mathrm{~h}$, followed by incubation with primary antibodies against FoxM1 (1:100; Bioworld Technology), E-cadherin (\#RLT1453, 1:100; Ruiying Biological) and vimentin ( \#RLT4879, 1:100; Ruiying Biological) overnight at $4^{\circ} \mathrm{C}$. Cells were washed with PBS for $15 \mathrm{~min}(3 \times 5 \mathrm{~min})$ and then incubated with the 2-step Plus Poly-HRP Anti-Mouse/Rabbit IgG detection system. Subsequently, after rinsing with PBS, the cells were stained with DAB and hematoxylin solution was used to stain the nuclei. The cells were imaged and analyzed under a microscope (Leica Microsystems).

Statistical analysis. All statistical analyses were performed using the SPSS software, version 22.0 (IBM Corp., Armonk, NY, USA). Student's t-test or ANOVA were performed to analyze the associations between FoxM1 expression and the clinicopathological features of the HSCC patients, as well as the results of the in vitro experiments, while a Pearson's correlation analysis was performed to evaluate the correlation between FoxM1 expression and Ki-67 expression in HSCC patients. Kaplan-Meier analysis was used to estimate overall or recurrence-free survival times; the differences between the survival curves were analyzed using the log-rank test. Univariate and multivariate analyses were performed using the Cox proportional hazards model. In all analyses, $\mathrm{P}<0.05$ was considered to indicate a statistically significant result. Continuous data are presented as the mean \pm standard deviation.

\section{Results}

FoxM1 is highly expressed in HSCC and is associated with tumor progression. To evaluate the expression of FoxM1 in HSCC, IHC was used to examine FoxM1 expression in 63 HSCC specimens at different levels of malignancy and 20 adjacent normal tissue specimens. FoxM1 was expressed predominantly in the nuclei, and mixed nuclear and cytoplasmic expression was also observed in some tumor cells. Notably, all HSCC tissues showed positive FoxM1 staining, by contrast, all of the adjacent tissues showed negative or weak staining of FoxM1 ( $\mathrm{P}<0.001$; Fig. 1A-E). In addition, fresh tissue samples from 7 HSCC patients [consisting of 2 earlystage (designated T1-2), 5 advanced-stage (designated T3-7) and 2 para-cancer samples (designated N1-2)] were collected to examine the protein and mRNA expression levels of FoxM1. The results showed that the advanced-stage tumors exhibited higher expression levels of FoxM1 compared with the earlystage tumors and para-cancer tissues (Fig. 1F and G).

To investigate potential associations between the expression level of FoxM1 and the clinicopathological characteristics of the HSCC patients, we used a Student's t-test or an ANOVA to compare the mean expression levels of FoxM1 between patients grouped according to their clinicopathological characteristics. The mean FoxM1 expression levels (IHC final scores) were significantly increased in poorly differentiated vs. welldifferentiated tumors $(\mathrm{P}=0.004)$, in tumor size $>2$ vs. $\leq 2 \mathrm{~cm}$ $(\mathrm{P}=0.002)$, in tumors of clinical stage III-IV vs. I-II $(\mathrm{P}=0.001)$, in cases with vs. without lymph node metastasis $(\mathrm{P}=0.002)$, 
A

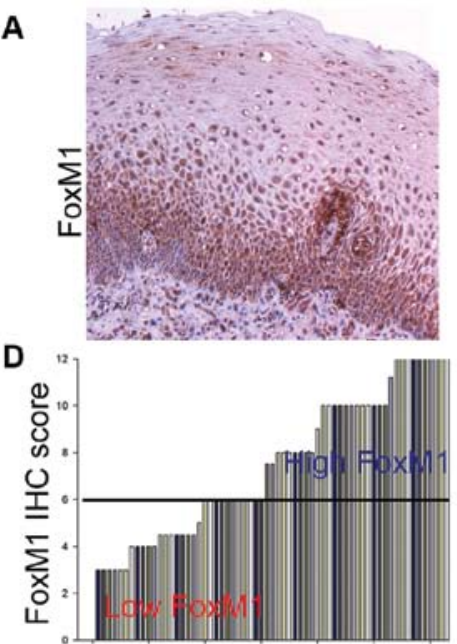

Patients, $\mathrm{N}=63$
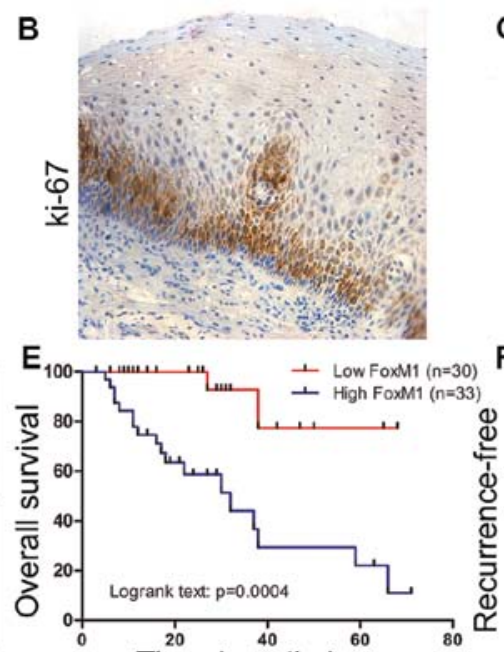

Time (months)
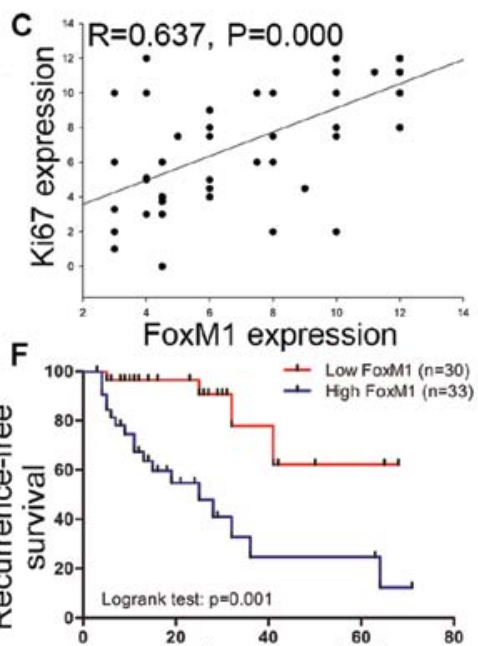

Time (months)

Figure 2. A high expression level of FoxM1 predicts poor prognosis in HSCC. (A and B) Representative immunohistochemical staining of FoxM1 compared with that of Ki-67 in HSCC tumor tissue. (A) FoxM1 (magnification, x100) and (B) Ki-67 (magnification, x100). (C) Relationship between Ki-67 and FoxM1 expression in HSCC ( $\mathrm{r}=0.637, \mathrm{P}<0.001$, Pearson's correlation coefficient). (D) Distribution of FoxM1 expression in 63 HSCC patients. (E and F) Kaplan-Meier curves for overall survival and recurrence-free survival times of HSCC patients $(\mathrm{P}=0.0004$ and $\mathrm{P}=0.001, \log$-rank test).

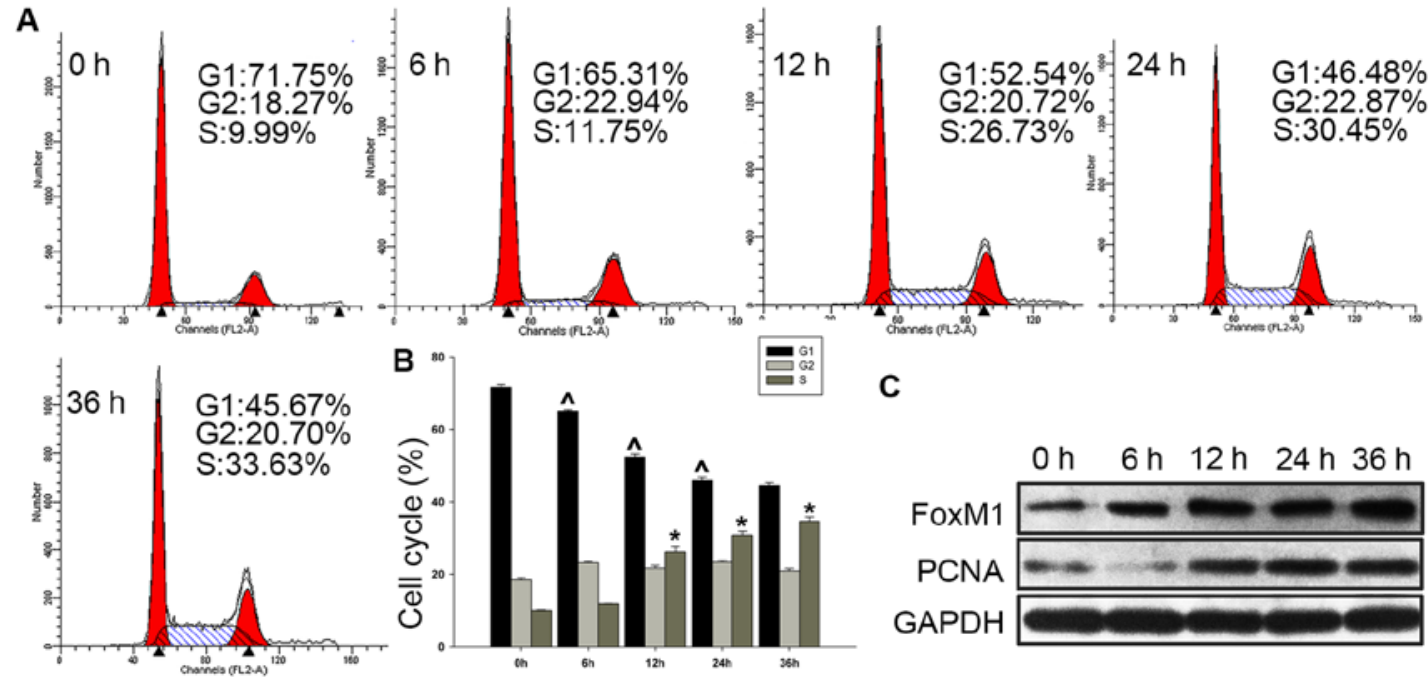

Figure 3. Expression of FoxM1 in proliferating Fadu cells. (A and B) Fadu cells were serum-deprived for 72 h in advance. (A) Cell cycle distribution was analyzed by flow cytometry. (B) Statistical analysis revealed a gradual decrease in the proportion of cells in G1 phase, and an increase in cells in S phase. (C) As more cells transitioned into $\mathrm{S}$ phase, the protein levels of FoxM1 and PCNA were also increased. Data represent the mean \pm standard deviation from three independent experiments. Data were analyzed with a Student's t-test. ${ }^{\wedge}$ " $\mathrm{P}<0.05$.

and according to the treatment method (surgery vs. surgery + radiation; surgery, radiation + chemoradiotherapy; and other treatment, $\mathrm{P}=0.045$ ), whereas there were no significant differences associated with patient's age or sex (Table I). All of these results indicate that FoxM1 is highly expressed in HSCC tissues, and that the overexpression of FoxM1 is involved in the degree of tumor malignancy in HSCC.

Elevated FoxM1 expression in HSCC patients is associated with poor prognosis. $\mathrm{Ki}-67$, a nuclear protein, is widely utilized as a proliferation marker in tumor specimens, and the Ki-67 index has been reported to be prognostic in various malignancies (21). Our results showed that there was a positive correlation between FoxM1 and Ki-67 expression (Pearson's correlation coefficient: $\mathrm{r}=0.637, \mathrm{P}<0.001$; Fig. 2A-C). Furthermore, we divided 63 patients with hypopharyngeal cancer into two groups, the results of the Kaplan-Meier analysis and log-rank test showed that patients with high FoxM1 expression $(\mathrm{N}=33$, FoxM1 IHC score $>6)$ had shorter overall survival $(\mathrm{P}=0.0004)$ and recurrence-free survival $(\mathrm{P}=0.001)$ times compared with those patients with low FoxM1 expression ( $\mathrm{N}=30$, FoxM1 IHC score $\leq 6)$ (Fig. 2D-F).

In addition, a Cox proportional hazards model was applied to estimate the effect of FoxM1 expression on HSCC patient survival. Univariate Cox regression analysis identified treatment method and the expression of FoxM1 and Ki-67 as significant prognostic factors. Using multivariate analysis, FoxM1 (HR, 5.051; 95\% CI, 1.079-23.262; P=0.038), Ki-67 
Table II. Univariate and multivariate analysis of overall survival.

\begin{tabular}{|c|c|c|c|c|c|c|}
\hline \multirow[b]{2}{*}{ Characteristics } & \multicolumn{3}{|c|}{ Univariate analysis } & \multicolumn{3}{|c|}{ Multivariate analysis } \\
\hline & HR & $95 \% \mathrm{CI}$ & P-value & HR & $95 \% \mathrm{CI}$ & P-value \\
\hline \multicolumn{7}{|l|}{ Age (years) } \\
\hline$<60 / \geq 60$ & 2.016 & $0.768-5.295$ & 0.155 & - & - & - \\
\hline \multicolumn{7}{|l|}{ Sex } \\
\hline Male/female & 21.895 & $0.001-890135.642$ & 0.569 & - & - & - \\
\hline \multicolumn{7}{|l|}{ Differentiation } \\
\hline High/poor & 1.642 & $0.629-4.285$ & 0.311 & 1.066 & $0.355-3.201$ & 0.909 \\
\hline \multicolumn{7}{|l|}{ Tumor size $(\mathrm{cm})$} \\
\hline$\leq 2 />2$ & 1.343 & $0.515-3.504$ & 0.547 & 0.146 & $0.027-0.796$ & $0.026^{\mathrm{a}}$ \\
\hline \multicolumn{7}{|l|}{ Clinical stage } \\
\hline I-II/III-IV & 3.442 & $0.795-14.910$ & 0.098 & 2.825 & $0.435-18.342$ & 0.277 \\
\hline \multicolumn{7}{|c|}{ Lymph node metastasis } \\
\hline Negative/positive & 2.171 & $0.785-6.001$ & 0.135 & 2.402 & $0.516-11.193$ & 0.264 \\
\hline \multicolumn{7}{|l|}{ Treatment } \\
\hline $\begin{array}{l}\text { Surgery only } \\
\text { Surgery and radia } \\
\text { Surgery, radiation } \\
\text { Other treatment }\end{array}$ & 1.883 & $1.182-3.000$ & $0.008^{\mathrm{b}}$ & 1.093 & $0.598-1.998$ & 0.771 \\
\hline \multicolumn{7}{|l|}{ Ki-67 expression } \\
\hline Low/high & 6.707 & $1.880-23.928$ & $0.003^{b}$ & 6.487 & $1.196-35.171$ & $0.030^{\mathrm{a}}$ \\
\hline \multicolumn{7}{|l|}{ FoxM1 expression } \\
\hline Low/high & 8.788 & $2.031-38.021$ & $0.004^{b}$ & 5.051 & $1.097-23.262$ & $0.038^{\mathrm{a}}$ \\
\hline
\end{tabular}

HR, hazard ratio; CI, confidence interval; ${ }^{a} \mathrm{P}<0.05,{ }^{\text {b }} \mathrm{P}<0.01$.

and tumor size were found to be independent prognostic factors for overall survival (Table II). Collectively, the results indicate that FoxM1 expression may act as an independent predictor for poor patient prognosis.

FoxM1 is highly expressed in proliferating Fadu cells. As the clinical data supported the hypothesis that high FoxM1 expression is associated with the proliferation of HSCC, further studies in the HSCC cell line Fadu were performed to verify this. We detected the expression of FoxM1 during cell cycle progression. Fadu cells were serum-deprived for $72 \mathrm{~h}$, and serum was then added to the cultures for 6,12 , 24 and $36 \mathrm{~h}$. It was observed that the proportion of cells in G1 phase decreased (from $71.58 \pm 0.76$ to $44.56 \pm 0.77 \%$ ), and those in $\mathrm{S}$ phase cells gradually increased (from $10.03 \pm 0.14$ to $34.57 \pm 1.24 \%$ ) with the addition of serum (Fig. 3A and B). In addition, the expression of FoxM1 was found to be increased concomitantly with the upregulation of the cell proliferation marker PCNA (Fig. 3C).

Knockdown of FoxM1 with siRNA blocks cell proliferation and induces cell cycle arrest in Fadu cells. To further identify the effect of FoxM1 on the biological functions of Fadu cells, cells were transfected with a negative control siRNA (si-NC) or one of four siRNAs targeting FoxM1, which were used to knockdown FoxM1 expression (Fig. 4A and B). The results showed that si-FoxM1\#2 was the most effective at decreasing FoxM1 mRNA expression (reduction of $74 \pm 13 \%$ ), and that si-FoxM1\#4 was moderately effective (reduction of $65 \pm 11 \%$ ); therefore, these two siRNAs were selected for use in subsequent experiments.

CCK-8 assays showed that FoxM1 knockdown resulted in a significant decrease in Fadu cell proliferation compared with the proliferation of si-NC-transfected cells $(\mathrm{P}<0.05$; Fig. 4C). Flow cytometry was used to analyze the effects of FoxM1 on cell cycle distribution, which revealed a reduced proportion of cells in $\mathrm{S}$ phase following treatment with si-FoxM1\#2 $(24.07 \pm 0.39 \% ; \mathrm{P}<0.05)$ or si-FoxM1\#4 $(29.15 \pm 1.03 \% ; \mathrm{P}<0.05)$ compared with si-NC treatment (34.84 $\pm 0.58 \%$; Fig. 4D and E). In addition, the protein expression levels of cyclin A1 and PCNA, which are closely related to cell proliferation, were also downregulated after treatment with si-FoxM1 (Fig. 4F and G) compared with si-NC treatment. Taken together, these data confirm that FoxM1 may regulate cell proliferation by influencing cell cycle progression.

Knockdown of FoxM1 with siRNA promotes apoptosis in Fadu cells. Annexin V/PI dual staining was used to confirm that FoxM1 knockdown could induce apoptosis in HSCC cells. As shown in Fig. 5A and B, after transfection with si-FoxM1 

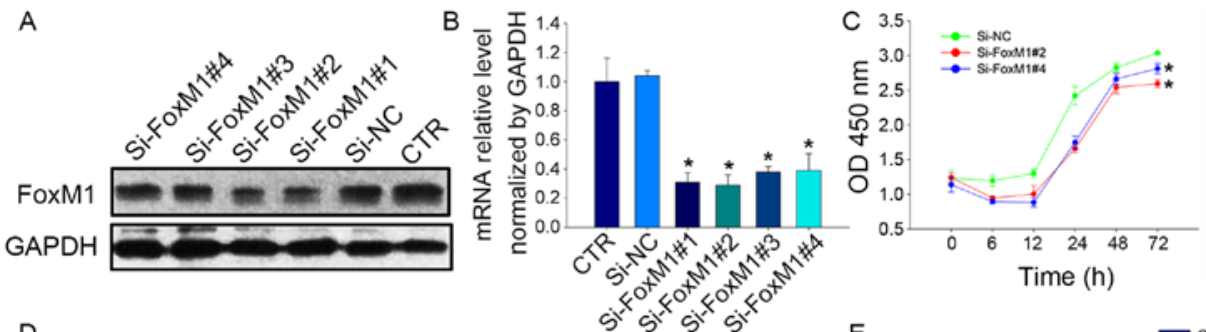

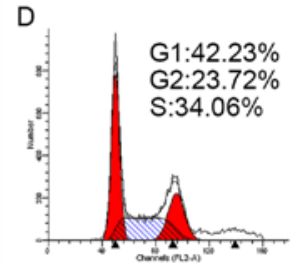

Si-NC

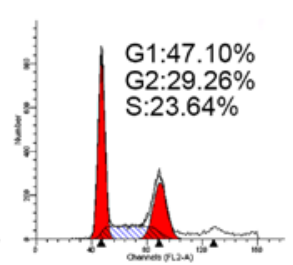

Si-FoxM1\#2

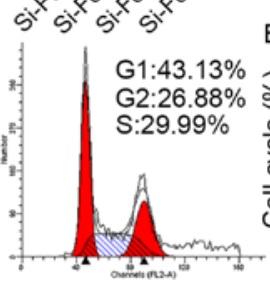

Si-FoxM1\#4
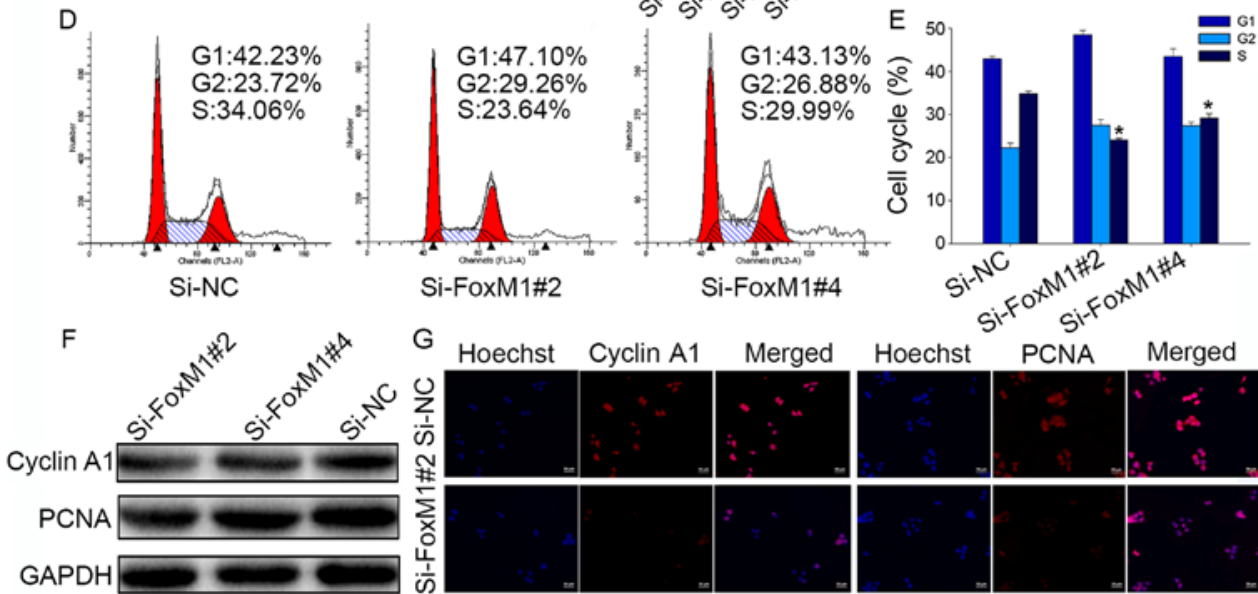

Figure 4. Knockdown of FoxM1 expression suppresses Fadu cell proliferation. (A and B) The relative protein and mRNA levels of FoxM1 in Fadu cells were significantly decreased by treatment with FoxM1 siRNAs, but were unaffected by si-NC treatment. (C) The CCK-8 assays showed that cell proliferation was inhibited with decreased FoxM1 expression ( $<<0.05$, t-test). (D and E) Flow cytometry showed that knockdown of FoxM1 expression in Fadu cells reduced the proportion of cells in S phase. (F and G) Protein expression of cyclin A1 and PCNA: (F) western blotting, (G) immunofluorescence staining (scale bars, $50 \mu \mathrm{m}) .{ }^{*} \mathrm{P}<0.05$ compared with si-NC.
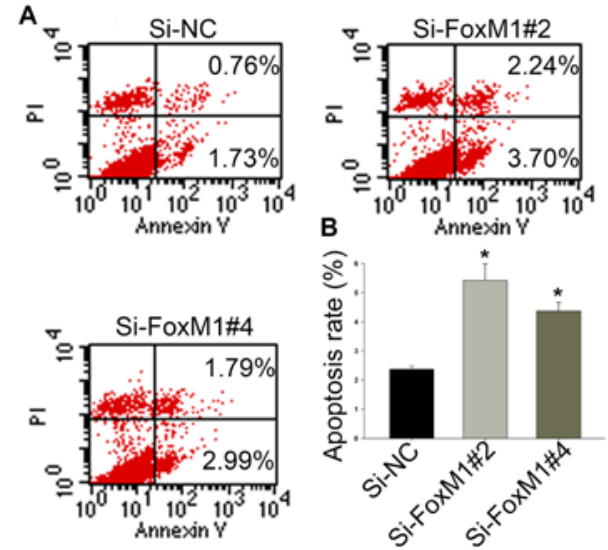

Figure 5. FoxM1-silencing induced cell apoptosis in Fadu cells (A and B) Flow cytometry showed an increase in the rate of cell apoptosis in Fadu cells following their treatment with FoxM1 siRNAs. Data are shown as the mean \pm standard deviation from three independent experiments. ${ }^{*} \mathrm{P}<0.05$ compared with si-NC.

for $48 \mathrm{~h}$, the proportion of cells undergoing apoptosis was increased from $2.37 \pm 0.10$ (si-NC) to $4.37 \pm 0.29$ (si-FoxM1\#4) or $5.42 \pm 0.56$ (si-FoxM1\#2). These results indicate that higher levels of FoxM1 decrease the rate of cell apoptosis in HSCC.

FoxM1 enhances the migration of Fadu cells by promoting EMT. The migratory capacities of Fadu cells transfected with si-NC or si-FoxM1 were detected with a wound-healing assay. Following culture in serum-free medium for $48 \mathrm{~h}$, the migratory activities of cells in the si-FoxM1\#2 group were notably impaired compared with those of the si-NC group (Fig. 6A and
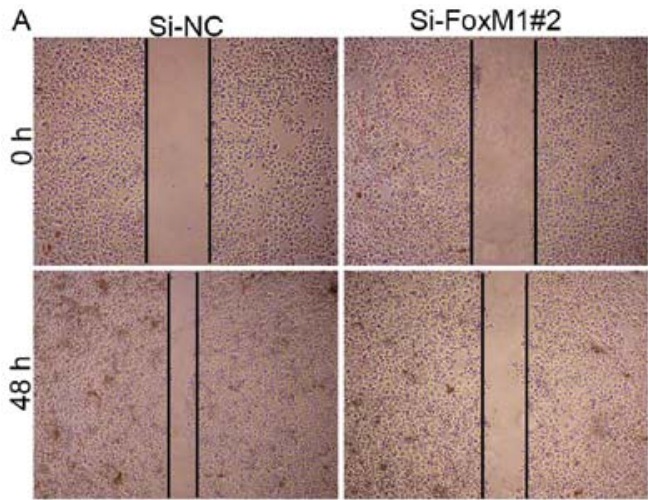

B

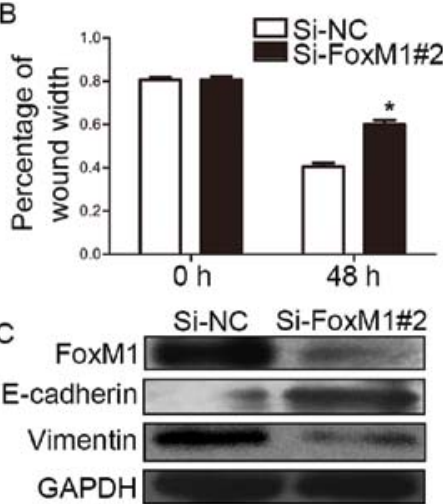

Figure 6. FoxM1 silencing inhibits the migration of Fadu cells. Cell migratory ability was analyzed with a wound-healing assay. (A) Representative images of the wound-healing assay (magnification, x200). (B) Relative migration distance of the cells; the amount of migrated cells and the relative migration distance were measured under a microscope. (C) Relative protein expression levels of EMT markers (E-cadherin and vimentin) following FoxM1 knockdown or si-NC treatment. ${ }^{*} \mathrm{P}<0.05$ compared with si-NC. 

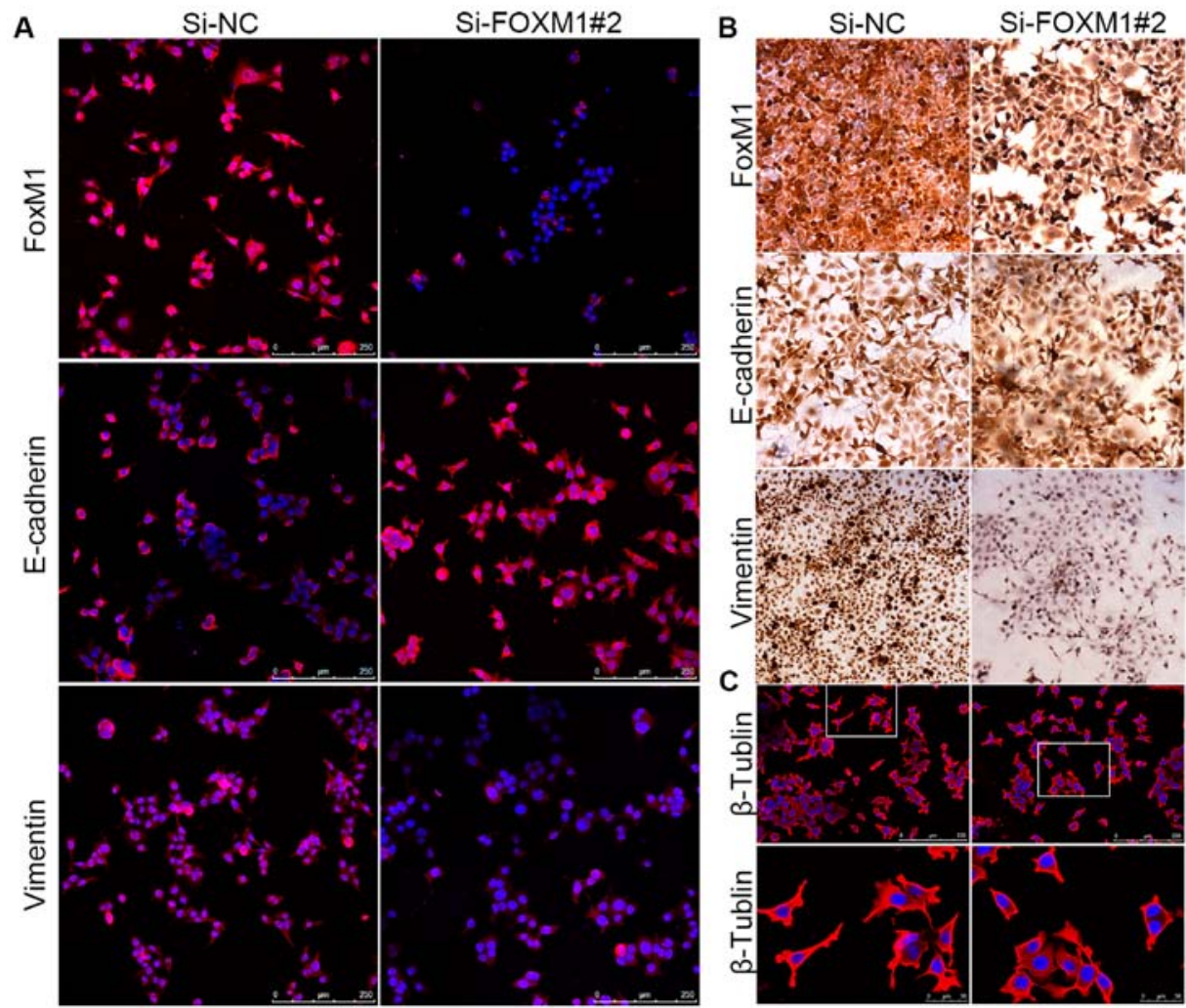

Figure 7. EMT marker expression is altered in Fadu cells following inhibition of FoxM1 expression. (A) Proteins were detected via immunofluorescence staining (scale bars, $250 \mu \mathrm{m}$ ) and (B) immunocytochemical staining (magnification, $\mathrm{x} 200$ ). (C) Representative images of $\beta$-tubulin expression observed via immunofluorescence staining (scale bars, 250 and $50 \mu \mathrm{m}$ ).

B). These results indicated that FoxM1 may stimulate cell migration in HSCC.

EMT, as an essential cell biological process related to embryonic development, also contributes to cancer metastasis and tumor progression (22). During EMT, proliferating epithelial cells acquire the features of a more invasive mesenchymal phenotype, including increased migratory activity and motility (23). In order to investigate whether FoxM1 contributes to EMT progression in HSCC cells, we evaluated the protein expression levels of FoxM1 and epithelial (E-cadherin) and mesenchymal (vimentin) markers in Fadu cells. Western blot analysis revealed that cells treated with si-FoxM1 exhibited increased E-cadherin expression and decreased vimentin expression (Fig. 6C). Consistent with the results of the western blot analysis, the data from the immunofluorescence assays showed a similar molecular expression pattern, indicating phenotypic changes between Fadu cells transfected with si-NC and si-FoxM1 (Fig. 7A). The results were also confirmed by immunocytochemical staining; following FoxM1 knockdown, the staining intensity of vimentin was decreased and the staining intensity of E-cadherin was increased (Fig. 7B). Collectively, these results indicated that FoxM1 overexpression could partially induce the transition to a mesenchymal phenotype, which may be a potential mechanism regulating migration in HSCC cells. Furthermore, using immunofluorescence microscopy, we detected the expression of $\beta$-tubulin, which plays an essential role in maintaining cell morphology and promoting the migration of Fadu cells. Cells treated with si-FoxM1 showed fewer fibers and pseudopodia compared with the si-NC group (Fig. 7C), indicating that FoxM1 may be involved in the formation and growth of invadopodia and, thus, the initiation of cell migration (24).

\section{Discussion}

The progression of carcinomas is affected by complex gene regulatory networks. At present, little has been established regarding the molecular mechanisms involved in HSCC. A previous study by Mochizuki et al (25) demonstrated that CD271 was a marker for tumor initiation and was associated with a poor prognosis in human HSCC. Additionally, STK33 was found to be a potential oncogene and a promising diagnostic marker for HSCC $(26,27)$. However, these two molecules alone do not explain the pathogenesis of HSCC.

Previous evidence has shown that aberrant expression of FoxM1 is involved in tumorigenesis and tumor progression $(9,28)$. Overexpression of FoxM1 has been shown to be associated with larger tumor size, lymph node metastasis, advanced tumor stage and poor disease-free and overall survival times in patients with estrogen receptor-positive breast cancer (29). In addition, patients with FoxM1-overexpressing angiosarcoma had significantly shorter disease-specific and event-free survival times compared with patients with low FoxM1 expression (30). Thus, a high level of FoxM1 in tumor patients appears to consistently indicate a high degree of malignancy and predict a poor prognosis. In the present study, FoxM1 was expressed to a significantly higher level in HSCC compared with para-tumor tissues in paraffin-embedded specimens. In addition, patients with advanced-stage HSCC had higher FoxM1 expression at the protein and mRNA 
levels compared with those patients with early-stage disease. Furthermore, analysis of the associations between FoxM1 expression and the clinicopathological features of 63 patients with HSCC revealed that patients defined as having poorly differentiated tumors, large tumor size, advanced-stage disease, positive lymph node metastasis and high Ki-67 expression exhibited higher FoxM1 expression. The results of KaplanMeier analysis and univariate and multivariate Cox regression analyses suggested that patients with high FoxM1 expression had a higher rate of mortality and tendency for recurrence. Collectively, these results indicate that high expression of FoxM1 may be a biological marker for malignant transformation and an indicator of poor prognosis in HSCC.

Abnormal proliferation has been demonstrated to be a key feature for cellular malignant transformation, and to play a vital role in tumorigenesis and development. FoxM1 is a typical proliferation-associated transcription factor involved in promoting the entry of cells into $\mathrm{S}$ phase and $\mathrm{M}$ phase, thereby maintaining the proper execution of mitosis $(7,31)$. Previous studies have reported that tumor cells with elevated FoxM1 expression showed high proliferative ability, whereas inhibition of FoxM1 expression led to the suppression of proliferative activity. It was shown that knockdown of FoxM1 expression significantly diminished NPC cell proliferation in vitro and inhibited the growth of NPC tumors in vivo (32). Furthermore, by using shRNA to diminish FoxM1 expression, Yang et al (33) found that reduced FoxM1 expression blocked the anchorage-independent growth and proliferation of MCF-7, MDA-MB-231 and ZR-75-30 breast cancer cells. In addition, FoxM1 was revealed to serve a pivotal role in tumor cell cycle progression. Previous studies also found that downregulation of FoxM1 inhibited cell proliferation and induced cell cycle arrest, with reduced the expression levels of cyclin B1, cyclin D1, and cyclin-dependent kinase 2 in clear cell renal cell carcinoma (34). Moreover, Jiang et al (18) found that thiostrepton-mediated downregulation of FoxM1 induced cell cycle arrest at $\mathrm{S}$ phase and inhibited DNA synthesis in LSCC cells in a dose- and time-dependent manner. Consistent with these prior studies, the present results demonstrated that Fadu cells with inhibited FoxM1 expression showed a decreased rate of proliferation and a reduced proportion of cells in S phase, along with decreased levels of PCNA and cyclin A1 expression, thus, confirming the role of FoxM1 in the regulation of tumor cell proliferation via its influence on cell cycle progression.

The survival and growth of cancer cells does not depend solely on proliferation, but also on resistance to cell death signals. FoxM1 was previously reported to act as an oncogene by regulating cell apoptosis (35). In the present study, we found that knockdown of FoxM1 expression markedly reduced the rate of Fadu cell apoptosis, validating the stimulatory effect of FoxM1 on HSCC aggressiveness.

Metastasis, which is regarded as a significant event during the malignant progression of a tumor, has been widely researched; however, its detailed mechanisms are not fully understood. Various evidence supports the hypothesis that FoxM1 plays an active role in tumor cell migration and metastasis in malignancies such as liver (36) and colorectal cancer (37). In the present study, we observed that FoxM1 overexpression was related to the metastasis of HSCC. Accumulating data have revealed that EMT leads to increased cell migration in several types of cancer (38). In colorectal cancer, EMT was found to be a key process in tumor metastasis $(37,39)$. In gastric cancer, it was demonstrated that Grhl2 reduced cell migration by inhibiting TGF- $\beta$-induced EMT in vitro and in vivo (40). In the present study, altered cell migratory capacity together with changes in the expression of EMT markers (E-cadherin and vimentin) in response to FoxM1 knockdown indicated that FoxM1 had a notable effect on the process of EMT in HSCC cells.

As one of the primary components and functional units of the cytoskeleton, $\beta$-tubulin is expressed in a wide variety of eukaryotic cells and is involved in invadopodium formation, which ultimately influences cell movement (41). We observed that Fadu cells transfected with si-NC had long erpseudopodia compared with those treated with si-FoxM1. Therefore, FoxM1-mediated EMT may be a pivotal step in HSCC metastasis.

There exist some limitations in this study. Firstly, due to low rates of HSCC, the number of samples is relatively small, with available follow-up data. Secondly, all of our samples were obtained from the same hospital. And then, the intricate mechanisms of FoxM1 in promoting HSCC progression need further studies to confirm. Furthermore, studies are also needed to enhance our results in vivo.

In conclusion, the present results demonstrated that FoxM1 was highly expressed in HSCC tissues, and that upregulated FoxM1 was significantly associated with the malignant phenotype and poor prognosis of HSCC patients, indicating a key role of FoxM1 in the regulation of HSCC progression. We also demonstrated that FoxM1 may serve as a promoter of HSCC cell cycle progression, thereby inducing cell proliferation. In addition, FoxM1 appeared to protect Fadu cells from apoptosis, and facilitate HSCC cell migration, possibly by promoting EMT. Collectively, these findings provide evidence for a novel molecular mechanism underlying the development of HSCC, and may lead to improvements in molecular targeted therapies for this type of tumor.

\section{Acknowledgements}

The present study was supported by the Scientific and Innovative Research Project of Nantong (MS32016015).

\section{References}

1. Torre LA, Bray F, Siegel RL, Ferlay J, Lortet-Tieulent J and Jemal A: Global cancer statistics, 2012. CA Cancer J Clin 65: 87-108, 2015

2. Cooper JS, Porter K, Mallin K, Hoffman HT, Weber RS Ang KK, Gay EG and Langer CJ: National Cancer Database report on cancer of the head and neck: 10-year update. Head Neck 31: 748-758, 2009.

3. Day D, Hansen AR and Siu LL: Hypopharyngeal cancer: looking back, moving forward. Curr Oncol: 23: 221-222, 2016.

4. Hall SF, Groome PA, Irish J and O'Sullivan B: The natural history of patients with squamous cell carcinoma of the hypopharynx. Laryngoscope 118: 1362-1371, 2008.

5. Hanahan D and Weinberg RA: The hallmarks of cancer. Cell 100: 57-70, 2000.

6. Martinez-Outschoorn UE, Peiris-Pages M, Pestell RG, Sotgia F and Lisanti MP: Cancer metabolism: A therapeutic perspective. Nat Rev Clin Oncol 14: 11-31, 2016.

7. Wierstra I: The transcription factor FOXM1 (Forkhead box M1): Proliferation-specific expression, transcription factor function, target genes, mouse models, and normal biological roles. Adv Cancer Res 118: 97-398, 2013. 
8. Petrovic V: FoxM1 regulates cell cycle progression through multiple mechanisms. University of Illinois at Chicago. Dissertations \& Theses - Gradworks, 2008.

9. Halasi M and Gartel AL: FOX(M1) news: it is cancer. Mol Cancer Ther 12: 245-254, 2013.

10. Miao L, Xiong X, Lin Y, Cheng Y, Lu J, Zhang J and Cheng N: Down-regulation of FoxM1 leads to the inhibition of the epithelial-mesenchymal transition in gastric cancer cells. Cancer Genet 207: 75-82, 2014.

11. Kong FF, Qu ZQ, Yuan HH, Wang JY, Zhao M, Guo YH, Shi J, Gong XD, Zhu YL, Liu F, et al: Overexpression of FOXM1 is associated with EMT and is a predictor of poor prognosis in non-small cell lung cancer. Oncol Rep 31: 2660-2668, 2014.

12. Jiang L, Wang P and Chen H: Overexpression of FOXM1 is associated with metastases of nasopharyngeal carcinoma. Ups J Med Sci 119: 324-332, 2014.

13. Sun HC, Li M, Lu JL, Yan DW, Zhou CZ, Fan JW, Qin XB, Tang HM and Peng ZH: Overexpression of Forkhead box M1 protein associates with aggressive tumor features and poor prognosis of hepatocellular carcinoma. Oncol Rep 25: 1533-1539, 2011.

14. Wu XR, Chen YH, Liu DM, Sha JJ, Xuan HQ, Bo JJ and Huang YR: Increased expression of forkhead box M1 protein is associated with poor prognosis in clear cell renal cell carcinoma. Med Oncol 30: 346, 2013.

15. Xia JT, Wang H, Liang LJ, Peng BG, Wu ZF, Chen LZ, Xue L, $\mathrm{Li} \mathrm{Z}$ and $\mathrm{Li} \mathrm{W}$ : Overexpression of FOXM1 is associated with poor prognosis and clinicopathologic stage of pancreatic ductal adenocarcinoma. Pancreas 41: 629-635, 2012.

16. Ahmad A, Wang Z, Kong D, Ali S, Li Y, Banerjee S, Ali R and Sarkar FH: FoxM1 down-regulation leads to inhibition of proliferation, migration and invasion of breast cancer cells through the modulation of extra-cellular matrix degrading factors. Breast Cancer Res Treat 122: 337-346, 2010.

17. Nakamura S, Hirano I, Okinaka K, Takemura T, Yokota D, Ono T, Shigeno K, Shibata K, Fujisawa S and Ohnishi K: The FOXM1 transcriptional factor promotes the proliferation of leukemia cells through modulation of cell cycle progression in acute myeloid leukemia. Carcinogenesis 31: 2012-2021, 2010

18. Jiang L, Wu X, Wang $\mathrm{P}$, Wen T, Yu C, Wei L and Chen $\mathrm{H}$ Targeting FoxM1 by thiostrepton inhibits growth and induces apoptosis of laryngeal squamous cell carcinoma. J Cancer Res Clin Oncol 141: 971-981,2015.

19. Huang C, Qiu Z, Wang L, Peng Z, Jia Z, Logsdon CD, Le X, Wei D, Huang $S$ and Xie K: A novel FoxM1-caveolin signaling pathway promotes pancreatic cancer invasion and metastasis. Cancer Res 72: 655-665, 2012.

20. Xue J, Lin X, Chiu WT, Chen YH, Yu G, Liu M, Feng XH, Sawaya R, Medema RH, Hung MC and Huang S: Sustained activation of SMAD3/SMAD4 by FOXM1 promotes TGF- $\beta$ dependent cancer metastasis. J Clin Invest 124: 564-579, 2014.

21. Ghanim B, Klikovits T, Hoda MA, Lang G, Szirtes I, Setinek U, Rozsas A, Renyi-Vamos F, Laszlo V, Grusch M, et al: Ki67 index is an independent prognostic factor in epithelioid but not in nonepithelioid malignant pleural mesothelioma: A multicenter study. Br J Cancer 112: 783-792, 2015.

22. Mego M, Reuben J and Mani S: Epithelial-mesenchymal transition (EMT) and cancer stem cells (CSCs): The Traveling Metastasis. In: Liquid Biopsies in Solid Tumors. Cancer Drug Discovery and Development. Cristofanilli M (eds). Humana Press, Totowa, NJ, pp67-80, 2017.

23. Chen T, You Y, Jiang H and Wang ZZ: Epithelial-mesenchymal transition (EMT): A biological process in the development, stem cell differentiation, and tumorigenesis. J Cell Physiol: Jan 12 , 2017 (Epub ahead of print). https://doi.org/10.1002/jcp.25797.
24. Chodniewicz D and Klemke RL: Guiding cell migration through directed extension and stabilization of pseudopodia. Exp Cell Res 301: 31-37, 2004.

25. Mochizuki M, Tamai K, Imai T, Sugawara S, Ogama N, Nakamura M, Matsuura K, Yamaguchi K, Satoh K, Sato I, et al: CD271 regulates the proliferation and motility of hypopharyngeal cancer cells. Sci Rep 6: 30707, 2016.

26. Huang L, Chen C, Zhang G, Ju Y, Zhang J, Wang H and Li J: STK 33 overexpression in hypopharyngeal squamous cell carcinoma: Possible role in tumorigenesis. BMC Cancer 15: 13, 2015.

27. Chen C, Huang L, Zhang G, Li Y, Li L, Bai X, Liu W, Wang H and Li J: STK33 potentiates the malignancy of hypopharyngeal squamous carcinoma: Possible relation to calcium. Cancer Biol Ther 17: 976-984, 2016.

28. Wierstra I: FOXM1 (Forkhead box M1) in tumorigenesis: Overexpression in human cancer, implication in tumorigenesis, oncogenic functions, tumor-suppressive properties, and target of anticancer therapy. Adv Cancer Res 119: 191-419, 2013.

29. Ahn H, Sim J, Abdul R, Chung MS, Paik SS, Oh YH, Park CK and Jang K: Increased expression of forkhead box M1 is associated with aggressive phenotype and poor prognosis in estrogen receptor-positive breast cancer. J Korean Med Sci 30: 390-397, 2015.

30. Ito T, Kohashi K, Yamada Y, Iwasaki T, Maekawa A, Kuda M, Hoshina D, Abe R, Furue M and Oda Y: Prognostic significance of forkhead Box M1 (FOXM1) expression and antitumor effect of FOXM1 inhibition in angiosarcoma. J Cancer 7: 823-830, 2016.

31. Wierstra I and Alves J: FOXM1, a typical proliferation-associated transcription factor. Biol Chem 388: 1257-1274, 2007.

32. Chen H, Yang C, Yu L, Xie L, Hu J, Zeng L and Tan Y: Adenovirus-mediated RNA interference targeting FOXM1 transcription factor suppresses cell proliferation and tumor growth of nasopharyngeal carcinoma. J Gene Med 14: 231-240, 2012.

33. Yang C, Chen H, Yu L, Shan L, Xie L, Hu J, Chen T and Tan Y: Inhibition of FOXM1 transcription factor suppresses cell proliferation and tumor growth of breast cancer. Cancer Gene Ther 20: 117-124, 2013.

34. Xue Y, Zhang G, Xu R, Zou X, Zhong X, Wu G, Wang X, Long D, $\mathrm{Wu} \mathrm{Y,Xu} \mathrm{H,et} \mathrm{al:} \mathrm{Overexpression} \mathrm{of} \mathrm{FoxM1} \mathrm{is} \mathrm{associated} \mathrm{with}$ tumor progression. J Urol 189: e783, 2013.

35. Bhat UG, Halasi M and Gartel AL: Thiazole antibiotics target FoxM1 and induce apoptosis in human cancer cells. PLoS One 4: e5592-e5592, 2009.

36. Park HJ, Gusarova G, Wang Z, Carr JR, Li J, Kim KH, Qiu J, Park YD, Williamson PR, Hay N, et al: Deregulation of FoxM1b leads to tumour metastasis. EMBO Mol Med 3: 21-34, 2011

37. Zhang C, Wang Y, Feng Y, Zhang Y, Ji B, Wang S, Sun Y, Zhu C, Zhang D and Sun Y: Glil promotes colorectal cancer metastasis in a Foxm1-dependent manner by activating EMT and PI3K-AKT signaling. Oncotarget 7: 86134-86147, 2016.

38. Lamouille S, Xu J and Derynck R: Molecular mechanisms of epithelial-mesenchymal transition. Nat Rev Mol Cell Biol 15: 178-196, 2014.

39. Chen B, Zeng X, He Y, Wang X, Liang Z, Liu J, Zhang P, Zhu H, $\mathrm{Xu} \mathrm{N}$ and Liang S: STC2 promotes the epithelial-mesenchymal transition of colorectal cancer cells through AKT-ERK signaling pathways. Oncotarget 7: 71400-71416, 2016.

40. Xiang J, Fu X, Ran W and Wang Z: Grhl2 reduces invasion and migration through inhibition of TGF $\beta$-induced EMT in gastric cancer. Oncogenesis 6: e284, 2017.

41. Ganguly A and Cabral F: The arresting action of microtubules in cell motility. Cell Cycle 10: 2614-2615, 2011. 